\title{
Construction of an 11-microRNA-based signature and a prognostic nomogram to predict the overall survival of head and neck squamous cell carcinoma patients
}

Yusheng Huang ${ }^{1 \dagger}$, Zhiguo Liu ${ }^{2 \dagger}$, Limei Zhong ${ }^{3}$, Yi Wen ${ }^{1}$, Qixiang Ye ${ }^{4}$, Donglin Cao ${ }^{3}$, Peiwu Li ${ }^{* *}$ and Yufeng Liu ${ }^{1,5^{*}}$

\begin{abstract}
Background: Head and neck squamous cell carcinoma (HNSCC) is a fatal malignancy owing to the lack of effective tools to predict overall survival (OS). MicroRNAs (miRNAs) play an important role in HNSCC occurrence, development, invasion and metastasis, significantly affecting the OS of patients. Thus, the construction of miRNA-based risk signatures and nomograms is desirable to predict the OS of patients with HNSCC. Accordingly, in the present study, miRNA sequencing data of 71 HNSCC and 13 normal samples downloaded from The Cancer Genome Atlas (TCGA) were screened to identify differentially expressed miRNAs (DEMs) between HNSCC patients and normal controls. Based on the exclusion criteria, the clinical information and miRNA sequencing data of 67 HNSCC samples were selected and used to establish a miRNA-based signature and a prognostic nomogram. Forty-three HNSCC samples were assigned to an internal validation cohort for verifying the credibility and accuracy of the primary cohort. Gene Ontology (GO) and Kyoto Encyclopedia of Genes and Genomes (KEGG) analyses were performed to explore the functions of 11 miRNA target genes.

Results: In total, 11 DEMs were successfully identified. An 11-miRNA risk signature and a prognostic nomogram were constructed based on the expression levels of these 11 DEMs and clinical information. The signature and nomogram were further validated by calculating the C-index, area under the curve (AUC) in receiver-operating characteristic curve analysis, and calibration curves, which revealed their promising performance. The results of the internal validation cohort shown the reliable predictive accuracy both of the miRNA-based signature and the prognostic nomogram. GO and KEGG analyses revealed that a mass of signal pathways participated in HNSCC proliferation and metastasis.
\end{abstract}

Conclusion: Overall, we constructed an 11-miRNA-based signature and a prognostic nomogram with excellent accuracy for predicting the OS of patients with HNSCC.

Keywords: microRNA, Head and neck squamous cell carcinoma, Overall survival, Risk signature, Nomogram

\footnotetext{
*Correspondence: doctorlipw@gzucm.edu.cn; wenrenlyf2008@163.com

${ }^{\dagger}$ Yusheng Huang and Zhiguo Liu are co-first author.

'The First Affiliated Hospital, Guangzhou University of Chinese Medicine, No.

12 Airport Road, Baiyun District, Guangzhou 510407, China

Full list of author information is available at the end of the article
}

(c) The Author(s). 2020 Open Access This article is licensed under a Creative Commons Attribution 4.0 International License, which permits use, sharing, adaptation, distribution and reproduction in any medium or format, as long as you give appropriate credit to the original author(s) and the source, provide a link to the Creative Commons licence, and indicate if changes were made. The images or other third party material in this article are included in the article's Creative Commons licence, unless indicated otherwise in a credit line to the material. If material is not included in the article's Creative Commons licence and your intended use is not permitted by statutory regulation or exceeds the permitted use, you will need to obtain permission directly from the copyright holder. To view a copy of this licence, visit http://creativecommons.org/licenses/by/4.0/ The Creative Commons Public Domain Dedication waiver (http://creativecommons.org/publicdomain/zero/1.0/) applies to the data made available in this article, unless otherwise stated in a credit line to the data. 


\section{Background}

Head and neck squamous cell carcinoma (HNSCC), the sixth most common and eighth most fatal malignancy worldwide [1] is an epithelial tumor arising from the oral cavity, nasal cavity, larynx, hypopharynx, and pharynx. Excessive consumption of tobacco and alcohol is considered a major risk factor for the occurrence and development of HNSCC [2]. In addition, human papillomavirus infection was recently confirmed as an important factor underlying HNSCC progression $[2,3]$. Despite the rapid development in examination techniques, HNSCC is generally detected at advanced stages owing to the lack of awareness of regular inspections and no or mild symptoms at early stages. Hence, HNSCC is associated with high mortality [4]. Many patients with HNSCC develop distant metastases within 5 years of receiving comprehensive and systematic chemotherapy [2]. This serves as a significant contributor to death. Thus, improvement in the screening rate of early tumors may be useful as an effective measure to reduce HNSCC-related mortality.

MicroRNAs (miRNAs) are short nonprotein-coding RNAs involved in post-transcriptional regulation of protein-coding gene expression via binding to the 3 '-untranslated regions of target mRNAs [5]. miRNAs participate in various physiological and pathological activities in the human body, including cell development, differentiation, cycle regulation, and apoptosis [6, 7]. Several studies have reported the potential diagnostic or prognostic roles of miRNAs in HNSCC, including those of miR-let-7a-5p, miR-3928, miR-936, miR-383, miR-615, miR-877, miR-9-5p, and $m i R-9-3 p$ [8-11]. The suppression of miR-30a and miR-379 expression could facilitate the oncogenic activity via upregulating the $D N M T 3 B$ expression and activating the hypermethylation of $A D H F E 1$ and $A L D H 1 A$ genes in oral squamous cell carcinoma [12]. In addition, circ0000495 has been shown to sponge $m i R-488-3 p$ expression and epigenetically silence TROP2 expression, resulting in the weakening of the proliferative capacity of HNSCC [13]. Thus, the functions of miRNAs affect HNSCC generation, development, and metastasis and are highly associated with the overall survival (OS) of patients with HNSCC.

In the present study, we investigated the miRNAs that were closely bound up with the OS of patients with HNSCC. A miRNA-based signature based on differentially expressed miRNAs (DEMs) as well as a novel miRNAbased prognostic model were constructed to reliably predict the OS of HNSCC patients and provide an important tool for clinicians to improve treatment regimens.

\section{Results}

Identification of DEMs associated with HNSCC patients

Raw HNSCC datasets, consisting of 71 HNSCC samples and 13 normal samples, were downloaded from The Cancer Genome Atlas (TCGA) database. In total, 797 miRNAs were acquired after eliminating those with expression levels
$<1$. In the heatmap (Fig. 1), the expression levels of 50 miRNAs were visually displayed. The differential expression of 797 miRNAs was visually observed using a volcano plot (Fig. 1). Of these, 90 miRNAs with $|\log 2 \mathrm{FC}| \geq 2$ and an adjusted $P$-value $<0.05$, including 54 upregulated and 36 downregulated miRNAs, showed significant differential expression. After eliminating the miRNAs detected in 13 normal samples and 4 patients, 90 DEMs were subjected to a univariate Cox proportional hazard regression (CPHR) analysis to determine the independent prognostic impact of individual genes. The results of the univariate CPHR analysis showed that 16 DEMs had the capacity to influence prognosis. Next, these 16 DEMs were subjected to LASSO Cox analysis, and a LASSO Cox regression model with a 10 -fold cross validation result was proposed (Fig. 1c and d). In total, 11 DEMs were identified the close correlation with the prognosis of patients with HNSCC (Table 1).

\section{Construction of a risk signature}

The 11 DEMs verified from the LASSO regression analysis were used to generate a risk signature as per the following formula: Risk score $=(0.236 \times$ expression $m i R-204-5 p)+(0.059 \times$ expression miR-499a-5p $)+(0.212 \times$ expression miR-498-5p $)-(0.062 \times$ expression miR-155-3p $)+$ $(0.434 \times$ expression miR-4714-3p $)-(0.141 \times$ expression $m i R-365 a-5 p)+(0.321 \times$ expression miR-30a-5p $)+(0.123 \times$ expression miR-1-5p $)+(0.240 \times$ expression miR-548f-3p $)+$ $(0.196 \times$ expression $\quad$ miR-518a-3p $)-(0.140 \times$ expression miR-196b-5p). Patients with HNSCC were distributed into high-risk and low-risk groups according to the median of risk score value. The new heatmap generated (Fig. 2a) clearly revealed the differences in the expression levels of the 13 DEMs between high-risk and low-risk groups. Eight DEMs (miR-204-5p, miR-499a-5p, miR-498-5p, miR-4714$3 p, m i R-30 a-5 p, m i R-1-5 p, m i R-548 f-3 p$, and $m i R-518 a-3 p)$ in the primary and internal validation cohorts showed higher expression in the high-risk group than that in the low-risk group. Contrarily, $m i R-155-3 p$, $m i R-365 a-5 p$, and $m i R-196 b-5 p$ were overexpressed in the low-risk group, suggesting that they might might function as tumor suppressors. The survival status and risk score distribution analyses further demonstrated the high risk in the high-risk group (Fig. 2b and c). We established a prognostic nomogram associated with 11 DEMs (Fig. 2d) and found that miR-4714-3p, miR-30a-5p, and miR-548f-3p strongly affected the OS of patients.

\section{Estimation of the reliability of the risk signature}

To estimate the reliability of the risk signature established herein, a Kaplan-Meier survival analysis (Fig. 3a) was performed. The result of this analysis revealed the shorter OS for patients from the high-risk group than for those from the low-risk group both in the primary $(P=5.393 \mathrm{e}-06)$ and internal validation cohorts $(P=$ 


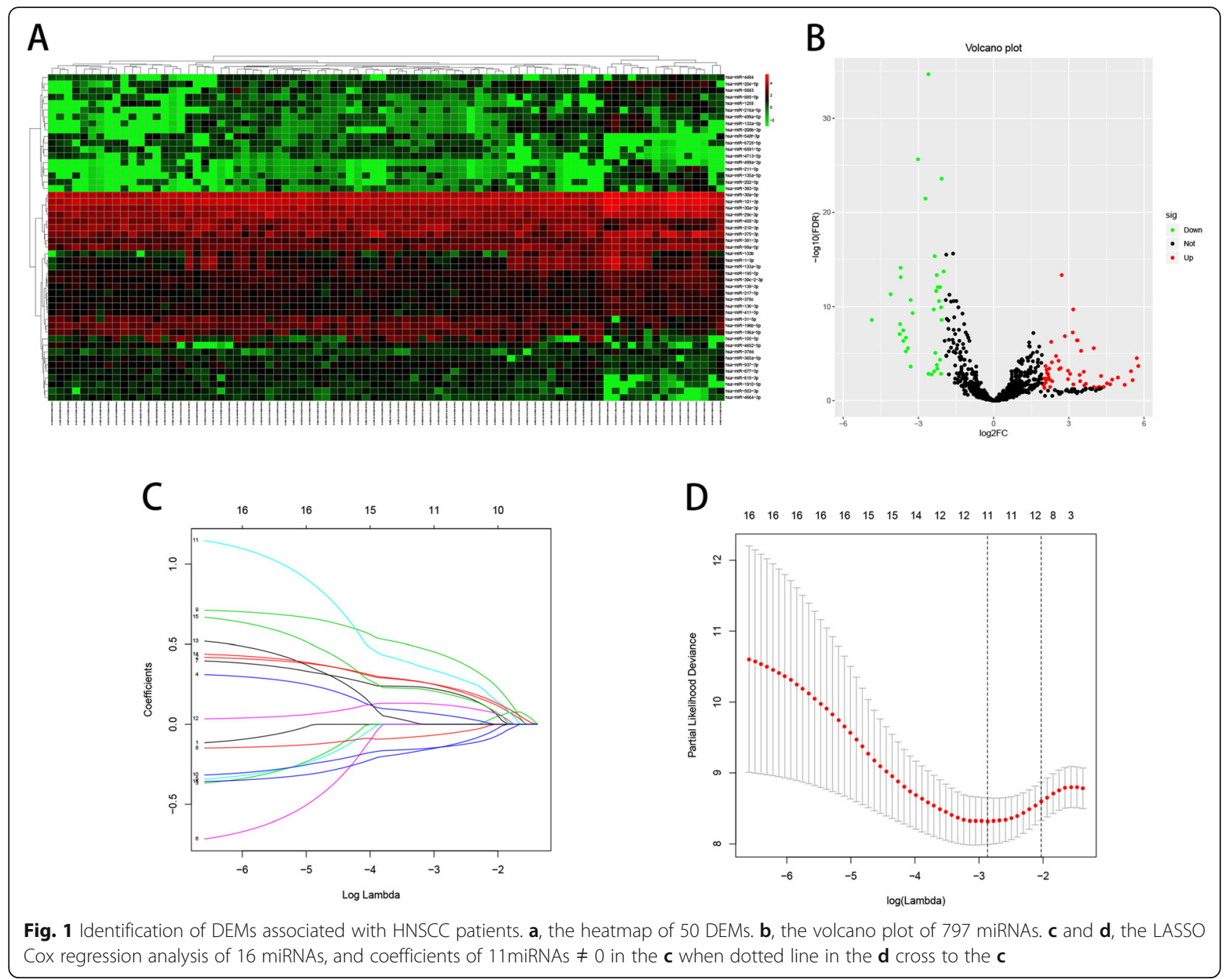

Table 1 LASSO regression analysis of miRNAs

\begin{tabular}{llll}
\hline miRNA & Coefficient & Type & Down-/upregulated \\
\hline hsa-miR-204-5p & 0.236292 & Risky & Up \\
hsa-miR-499a-5p & 0.059085 & Risky & Up \\
hsa-miR-498-5p & 0.211516 & Risky & Up \\
hsa-miR-155-3p & -0.061566 & Protective & Down \\
hsa-miR-4714-3p & 0.434481 & Risky & Up \\
hsa-miR-365a-5p & -0.141218 & Protective & Down \\
hsa-miR-30a-5p & 0.321480 & Risky & Up \\
hsa-miR-1-5p & 0.122628 & Risky & Up \\
hsa-miR-548f-3p & 0.240339 & Risky & Up \\
hsa-miR-518a-3p & 0.196145 & Risky & Up \\
hsa-miR-196b-5p & -0.140244 & Protective & Down \\
\hline
\end{tabular}

5.176e-04). In addition, the area under the curve (AUC) value of the risk signature for 5-year OS had reliable predictive accuracy (Fig. $3 \mathrm{~b}$ ). In the primary cohort, the AUC values of the receiver-operating characteristic (ROC) curve analysis for the risk signature for 1-, 3-, and 5-year OS were $0.802,0.804$, and 0.825 , respectively. These values were reported to be $0.724,0.811$, and 0.829 for 1-, 3-, and 5-year OS in the internal validation cohort (Fig. 3c). The calibration curves of the risk signature in the two cohorts revealed excellent agreement between the expected and actual outcomes for 3- and 5-year OS (Fig. 3d). Furthermore, the C-index value for both the primary and internal cohorts was 0.77 , indicating considerable accuracy.

\section{Establishment and evaluation of a nomogram}

The clinical information, including age, sex, TNM stage and grade, and hypoxia score, was remarkably associated with the OS of patients with HNSCC (Table 2). Univariate and multivariate CPHR analyses were carried out to 


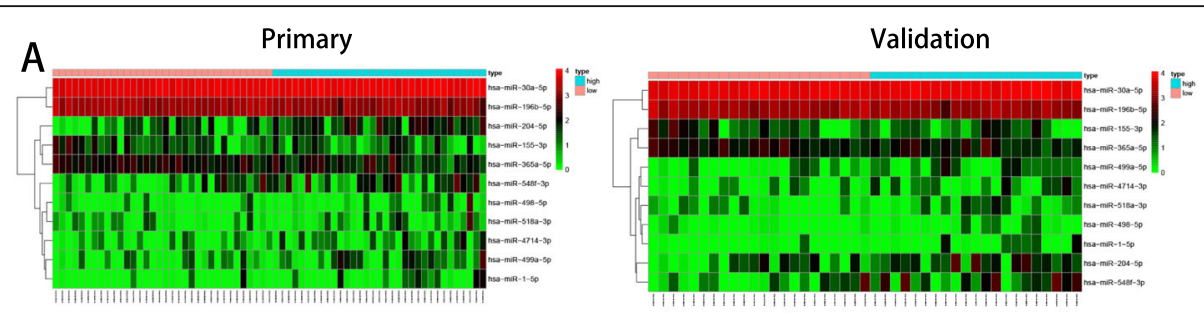

B
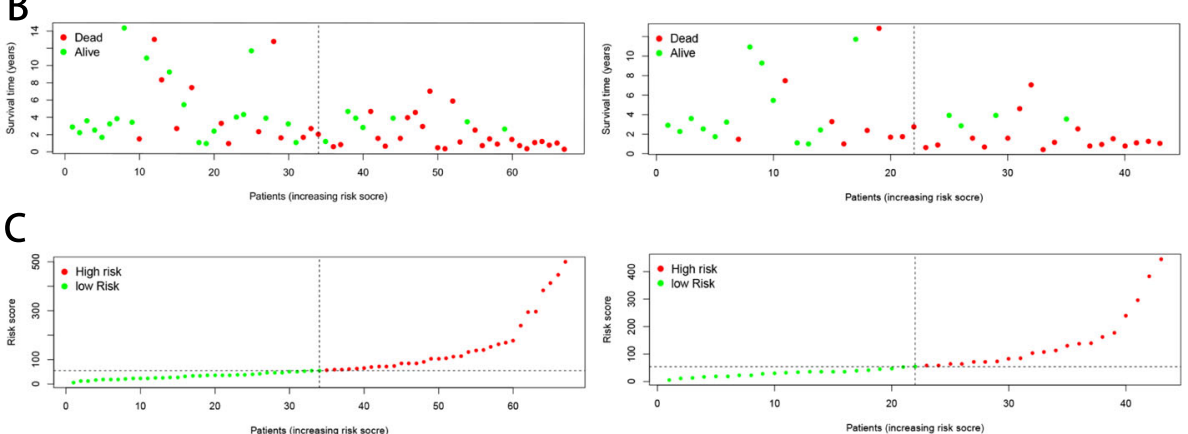

D

Points

hsa-miR-204-5p

hsa-miR-499a-5p

hsa-miR-498-5p

hsa-miR-155-3p

hsa-miR-4714-3p

hsa-miR-365a-5p

hsa-miR-30a-5p

hsa-miR-1-5p

hsa-miR-548f-3p

hsa-miR-518a-3p

hsa-miR-196b-5p

riskScore

risk

Total Points

3-Year Survival

5-Year survival

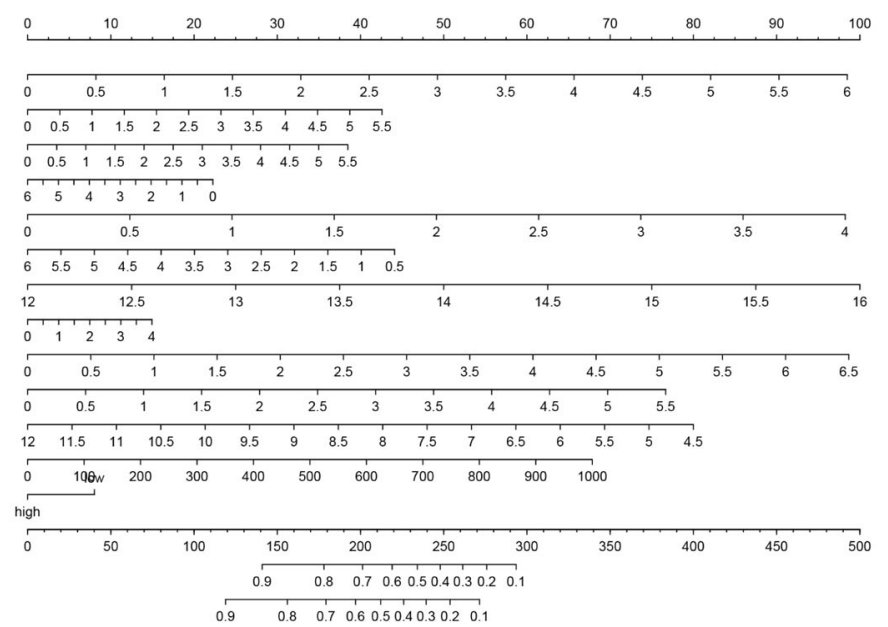

Fig. 211 miRNAs-based risk signature construction. a, the heatmap of 11 miRNAs. $\mathbf{b}$, the distribution of OS. $\mathbf{c}$, the distribution of risk score. $\mathbf{d}$, the prognostic nomogram based on risk signature and 11 miRNAs was used to predict 3- and 5-year OS of patients with HNSCC

obtain information and risk scores for the primary cohort (Fig. 4a and b). In the primary cohort, we proved those factors were independent prognostic variables of OS, including TNM stage, hypoxia score, and risk score. Further, a prognostic nomogram was established using three independent prognostic variables (Fig. 4c). The miRNA signature was more effective in predicting the OS of HNSCC patients, followed by TNM stage and hypoxia score. Further, the AUC values of the ROC curves of the two independent prognostic variables demonstrated that each variable had credible predictive accuracy, especially the miRNA signature (Fig. 5a). The AUC values of the ROC analysis for nomogram were $0.705,0.729$, and 0.827 at 1 -, 3-, and 5-year OS, respectively, for the primary cohort, and $0.723,0.748$, and 0.837 at 1-, 3-, and 5-year OS, respectively, for the internal validation cohort (Fig. 5b). To assess the calibration capability of this prognostic model, we established calibration curves and found that the predicted and actual survival in the two cohorts corresponded using this prognostic model (Fig. 5c). The $\mathrm{C}$-index values of the nomogram were 0.776 and 0.744 in the primary and internal validation cohorts, respectively.

\section{Target genes functional enrichment analysis}

We predicted the corresponding target genes using three independent databases to confirm the potential biological functions of the 11 DEMs. In total, 38,191 target genes were detected, of which 305 genes were overlapping. Thus, these overlapping genes potentially modulated by the 11 DEMs were subjected to GO and KEGG enrichment analyses. Based on the criterion of a $P$-value $<0.05,42$ categories, including nucleus, cytoplasm, and membrane, showed a 

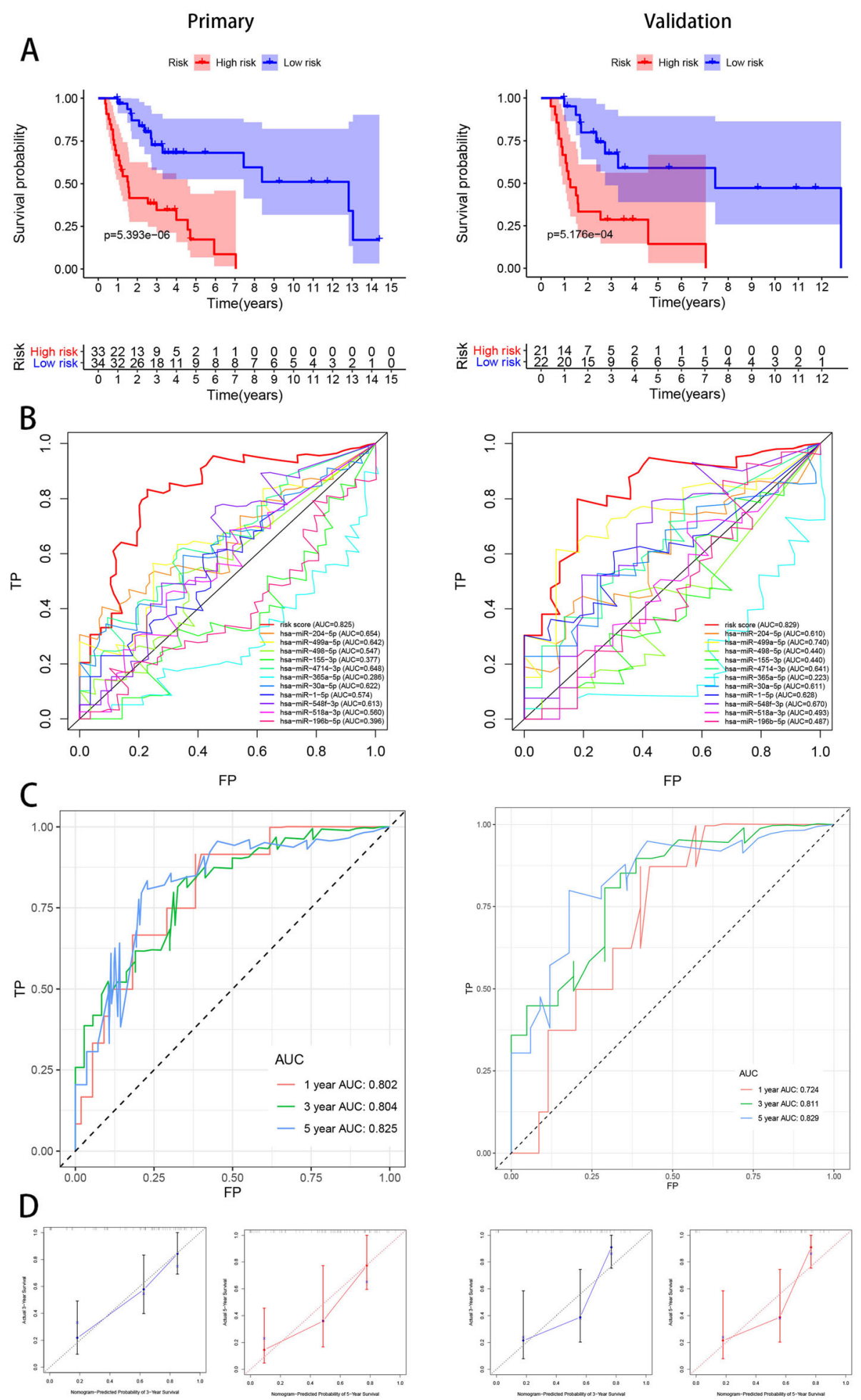

Fig. 311 miRNAs-based risk signature evaluation. a, the Kaplan-meier survival analysis revealed the difference of survival rate between high and low risk group. b, AUC in ROC analysis for 11 DEMs and risk signature at 5-years survival time. c, 1-, 3- and 5-year AUC in ROC analysis. d, Calibration curves of risk signature used for evaluating the 3- and 5- year AUC 
Table 2 Clinicopathologic characteristics of HNSCC patients in two cohorts

\begin{tabular}{|c|c|c|c|c|}
\hline \multirow[t]{2}{*}{ Variables } & \multicolumn{2}{|c|}{ Primary cohort } & \multicolumn{2}{|c|}{ Validation cohort } \\
\hline & $N=67$ & $\%$ & $N=43$ & $\%$ \\
\hline \multicolumn{5}{|l|}{ Age } \\
\hline$\leq 60$ & 23 & 34.33 & 14 & 32.56 \\
\hline$>60$ & 44 & 65.67 & 29 & 67.44 \\
\hline \multicolumn{5}{|l|}{ Sex } \\
\hline Female & 25 & 37.31 & 15 & 34.88 \\
\hline Male & 42 & 62.68 & 28 & 65.12 \\
\hline \multicolumn{5}{|l|}{ TNM stage } \\
\hline I & 2 & 2.99 & 2 & 4.65 \\
\hline$\|$ & 18 & 26.87 & 10 & 23.26 \\
\hline III & 10 & 14.93 & 7 & 16.28 \\
\hline IV & 32 & 47.76 & 21 & 48.84 \\
\hline NA & 5 & 7.46 & 3 & 6.98 \\
\hline \multicolumn{5}{|c|}{ neoplasm histologic grade } \\
\hline 1 & 12 & 17.91 & 9 & 20.93 \\
\hline 2 & 39 & 58.21 & 23 & 53.49 \\
\hline 3 & 14 & 20.90 & 10 & 23.26 \\
\hline NA & 2 & 2.99 & 1 & 2.33 \\
\hline \multicolumn{5}{|c|}{ Ragnum Hypoxia Score } \\
\hline$<0$ & 1 & 1.49 & 1 & 2.33 \\
\hline $0-9$ & 10 & 14.93 & 5 & 11.63 \\
\hline $10-19$ & 49 & 73.13 & 31 & 72.09 \\
\hline$\geq 20$ & 7 & 10.45 & 6 & 13.95 \\
\hline \multicolumn{5}{|c|}{ Survival status } \\
\hline Alive & 28 & 41.79 & 17 & 39.53 \\
\hline Dead & 39 & 58.21 & 26 & 60.47 \\
\hline
\end{tabular}

strong influence on the occurrence and development of cancer (Fig. 6a). In addition, the KEGG enrichment analysis revealed the multiple pathways that play key roles in HNSCC progression, especially the neurotrophin signaling pathway and protein processing in endoplasmic reticulum (Fig. 6b).

\section{Discussion}

Many reports have suggested the participation of miRNAs in different pathological processes related to HNSC $\mathrm{C}[8,13]$. However, there is no single representative miRNA that predicts the OS of patients with HNSCC. In the present study, we collected information on patients with HNSCC and evaluated their miRNA expression levels to systematically analyze the miRNAs and clinical characteristics associated with their OS.

We performed the CPHR and LASSO regression analyses and identified 11 DEMs, including miR-204-5p, miR-499a5p, miR-498-5p, miR-155-3p, miR-4714-3p, miR-365a-5p,
miR-30a-5p, miR-1-5p, miR-548f-3p, miR-518a-3p, and $m i R-196 b-5 p$, that were confirmed to influence the OS of patients with HNSCC. Kimura et al. conducted a microarray analysis and found that the expression of $m i R-30 a-5 p$ was upregulated both in HNSCC and esophagus squamous cell carcinoma cell lines compared to that in normal squamous epithelial cell lines [14]. Another study reported the significantly upregulated expression of $m i R-30 a-5 p$ and $m i R-769-$ $5 p$ in the plasma of patients with oral squamous cell carcinoma (OSCC) and their effectiveness as minimally invasive biomarkers for OSCC diagnosis [15]. However, the function of $m i R-30 a-5 p$ in patients with HNSCC is yet unclear. A previous study found that miR-769-5p promotes the growth of glioma cells through the suppression of KMT2A [16] and could be useful as a therapeutic target for glioma treatment. The expression of $m i R-518 a-3 p$ has been found to be upregulated in hepatocellular carcinoma, as demonstrated by Taqman low-density miRNA array and quantitative realtime polymerase chain reaction $[17,18]$, indicative of its tumor-inducing properties. In addition, miR-518a-3p has been shown to promote the proliferation, migration, and invasion of choriocarcinoma cells; knockdown of miR-518a$3 p$ expression has been found to induce $\mathrm{S}$ phase arrest in choriocarcinoma cells [19]. miR-155-3p expression downregulation in breast cancer is related to resistance to tumor invasion and metastasis and reduction in paclitaxel resistance [20]. miR-365a-5p inhibits the viability, colony formation, migration, and invasion of non-small cell lung cancer cells by negatively regulating Pellino E3 ubiquitin protein ligase family member 3 (PELI3) [21], and PELI3 silencing promotes the miR-365a-5p-mediated suppression of protumoral effects. However, the expression of miR-499a-5p was down-regulated in OSCC tissues [22]. During glioma progression, $m i R-499 a-5 p$ exerts tumor-suppressive effects by regulating the formation of vasculogenic mimicry [23]. In addition, the overexpression of miR-499a-5p can markedly inhibit tumor cell proliferation, invasion, and migration [24]. The expression of miR-204-5p is suppressed in HNSCC, wherein it serves as a tumor suppressor and inhibits tumor growth and metastasis [25]. miR-196b-5p expression upregulation in HNSCC has also been verified using TCGA database [26]. Although these authors described three different miRNAs, their study had fewer samples for verification or used different calculation methods to select miRNAs. Therefore, it was imperative to further verify the functions of these miRNAs. The miRNAs miR-498-5p, miR-1-5p, $m i R-548 f-3 p$, and $m i R-4714-3 p$ have not been reported in previous HNSCC-related studies; thus, additional research is warranted to determine their functions in HNSCC.

GO analysis revealed 11 DEMs that were primarily enriched at the nucleus, cytoplasm, and membrane and were related to the positive regulation of transcription from the RNA polymerase II promoter. These locations and pathways were associated with different physiological 


\begin{tabular}{|c|c|c|}
\hline$A$ & pvalue & Hazard ratio \\
\hline Age & 0.268 & $1.151(0.897-1.477)$ \\
\hline Sex & 0.753 & $0.902(0.473-1.717)$ \\
\hline TNM-Stage & 0.013 & $2.422(1.202-4.877)$ \\
\hline Grade & 0.254 & $1.288(0.834-1.990)$ \\
\hline Hypoxia Score & 0.006 & $1.557(1.137-2.134)$ \\
\hline riskScore & $<0.001$ & $1.008(1.005-1.011)$ \\
\hline
\end{tabular}

B

pvalue

Hazard ratio

Age

0.236

$1.203(0.887-1.631)$

Sex

0.551

$1.247(0.603-2.578)$

'TNM-Stage'

0.007

$2.630(1.302-5.310)$

Grade

0.915

$1.026(0.636-1.656)$

'Hypoxia Score` 0.031

1.424(1.032-1.964)

riskScore

$<0.001$

$1.009(1.005-1.012)$

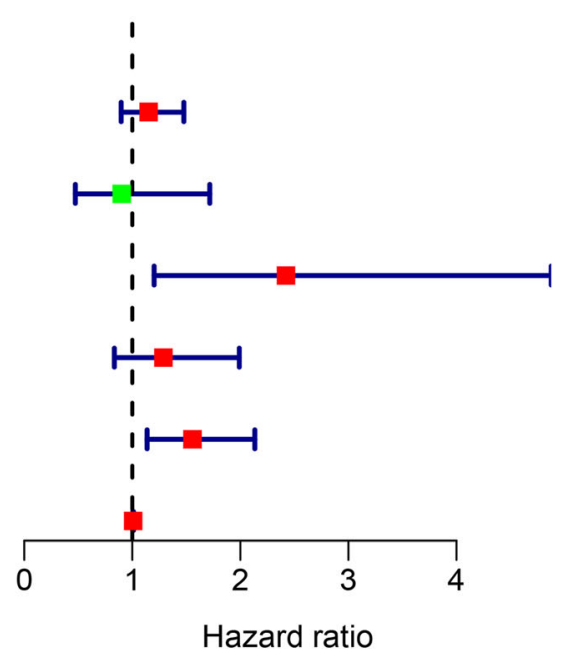

Hazard ratio

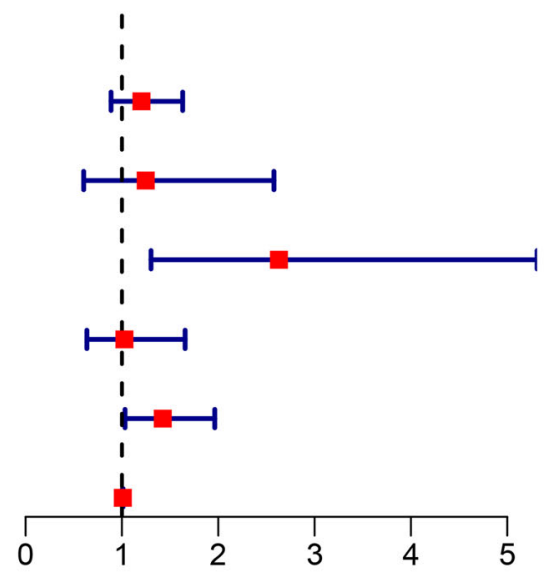

C

Hazard ratio

Points

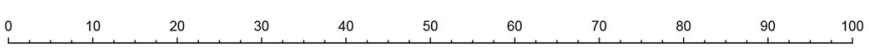

TNM-Stage

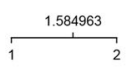

Hypoxia Score

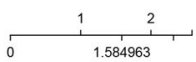

riskScore

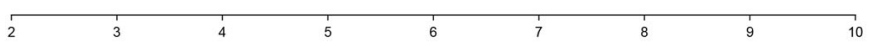

Total Points

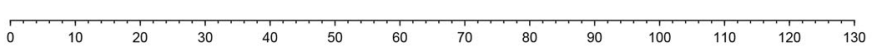

3-Year Survival

5-Year survival

Fig. 4 Univariate and multivariate analysis were used to verified factors related HNSCC patient. $\mathbf{a}$ and $\mathbf{b}$, the univariate CPHR analysis and the multivariate CPHR analysis were using to estimate whether these clinical factors and risk signature are independent prognostic variables or not. c, the prognostic nomogram established by risk signature, TMN-stage and hypoxia score was used to predict 3-and 5-year OS of patients with HNSCC 

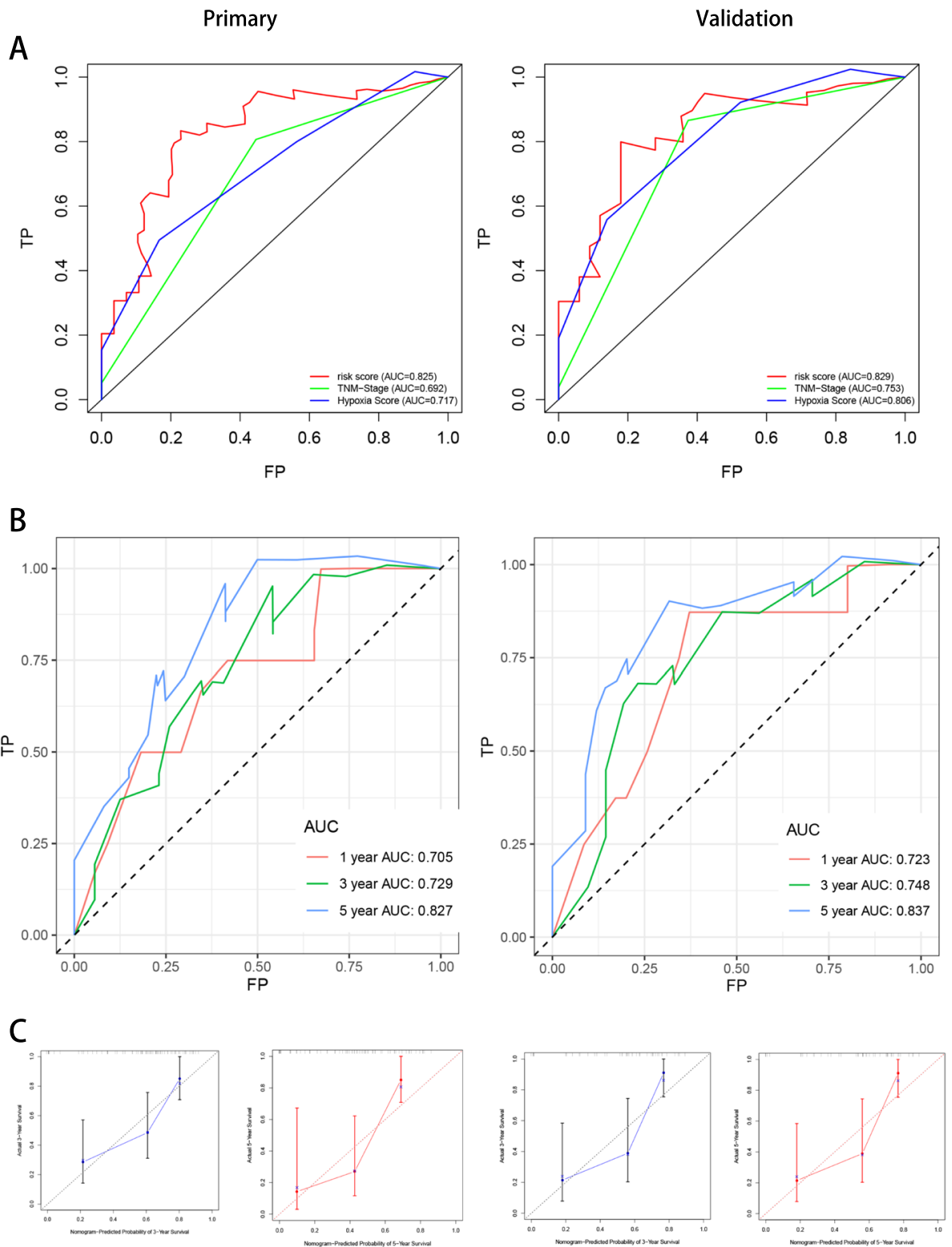

Fig. 5 The novel nomogram validation. a, AUC of risk signature, TMN-stage and hypoxia score in ROC analysis were calculated at 5-year survival time. $\mathbf{b}, \mathrm{AUC}$ of risk signature in ROC analysis were calculated at 1-, 3- and 5-years survival time. $\mathbf{c}$, Calibration curves of risk signature used for evaluating the 3- and 5- year AUC

and pathological activities of the human body. Uncontrolled disease activity accelerates the occurrence and development of HNSCC, highlighting the importance of pathological changes related to these locations and pathways. Furthermore, the KEGG enrichment analysis revealed that the neurotrophin signaling pathway, protein processing in the endoplasmic reticulum, and microRNAs in cancer significantly affected HNSCC occurrence and development. We found that several targeted genes exert their functions through the neurotrophin signaling pathway, which performs key functions in the occurrence and development of HNSCC by regulating cell survival, angiogenesis, tissue invasion, DNA damage resistance, and epithelial-to-mesenchymal transition [27-30]. Gkouveris et al. demonstrated that interferon-gamma inhibits OSCC cell viability and migration and increases cellular apoptosis through the endoplasmic reticulum stress pathway [31]. Further, dentin sialophosphoprotein induces OSCC cell viability [32] and HIV protease inhibitors with radiosensitizing activities mediate HNSCC cell apoptosis [33] through the activation of the endoplasmic reticulum stress mechanisms. Overall, these pathways predicted herein may play an important role in HNSCC development and progression. 


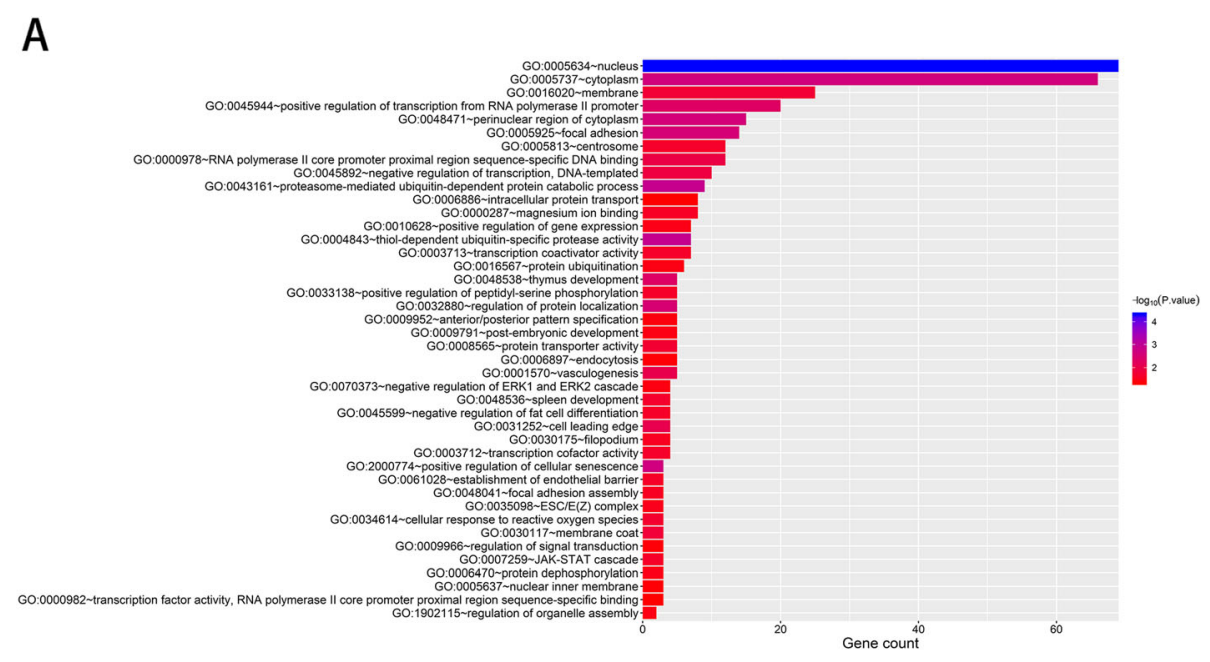

B

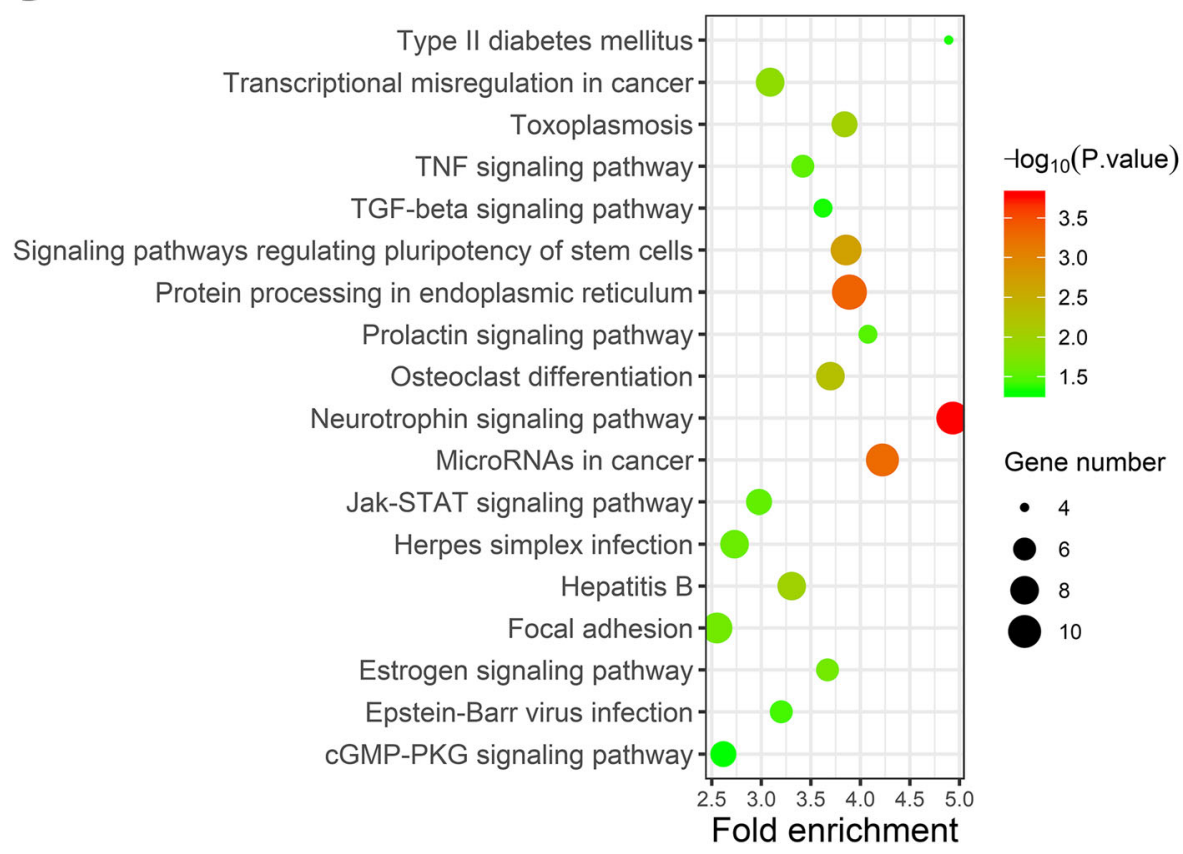

Fig. 6 Gene set enrichment analysis. a, GO enrichment analyses revealed 11 miRNAs potentially regulated HNSCC progression via 42 categories. b, KEGG enrichment analyses revealed 18 signal pathways taken part in HNSCC progression

In the present study, we analyzed the miRNA expression levels and clinical information of 71 HNSCC patients and 13 normal subjects. A mess of samples would effectively reduce bias. The univariate CPHR and LASSO regression analyses yielded 11 DEMs. Thus, we constructed and systematically validated the risk signature and nomogram, which showed high predictive accuracy. In comparison with invasive tests or imageological examination, the risk signature and predicted nomogram were convenient and economic but must be verified in clinical studies and cell or animal experiments.

\section{Conclusion}

We constructed an 11-miRNA risk signature and a nomogram that showed high correlation with HNSCC prognosis. The 3- and 5-year OS of patients with HNSC $\mathrm{C}$ could be accurately predicted through the risk signature and nomogram, thus serving as an effective tool for clinicians to facilitate decision-making.

\section{Methods}

Data resources and preprocessing

The miRNA sequencing data and related clinical information of patients with HNSCC were obtained from 
TCGA database (https://portal.gdc.cancer.gov/). The "edgeR" package in R software was used to identify DEMs from 797 miRNAs in 71 HNSCC samples and 13 normal samples based on $|\log 2 \mathrm{FC}| \geq 2$ and adjusted $P$-value < 0.05 . We then integrated the miRNA expression data and clinical information based on sample ID, and excluded four patients because they had no significant clinical date or their OS was less than 30 days to avoid introduction of mixed factors. Finally, 67 patients with HNSCC were enrolled as the primary cohort. In order to unbiasedly appraise the final model, 44 patients randomly selected from the primary cohort were designed as the internal validation cohort by using the "caret" package in R software.

\section{Construction of risk score formula}

DEMs were detected from the univariate CPHR analysis of the primary cohort. These DEMs with a $P$-value < 0.05 were subjected to LASSO CPHR analysis to produce a miRNA signature using the "glmnet" package in $\mathrm{R}$ software. The risk score of each patient relied on the miRNA expression level and the regression coefficient obtained from the LASSO regression analysis. The risk score was calculated as follows: Risk score (miRNAbased classifier $)=$ sum of coefficients $\times$ expression level of miRNAs, refer to previous description [34]. According to the median risk score value, 67 patients with HNSCC were divided into high-risk and low-risk groups.

\section{Assessment of miRNA signature}

The survival rate between the high- and low-risk groups were compared via the Kaplan-Meier survival analysis. A time-dependent ROC curve analysis performed using "survival ROC" packages estimated the sensitivity and specificity of each miRNA signature on the basis of the AUC value.

The differences in the signature between predicted survival and actual survival were assessed and calibration curves were constructed. The $\mathrm{C}$-index values were calculated to measure the predictive performance of the risk signature. All analyses were performed to compare the consistency between the primary and internal validated cohorts, refer to previous description [34].

\section{Construction and evaluation of a novel nomogram}

Due to the $\mathrm{T}$ stage, $\mathrm{M}$ stage, $\mathrm{N}$ stage exist serious information loss, those clinical informations were excluded for guaranteeing the reality of this study. Risk signature and other clinical factors, such as age, sex, neoplasm histologic grade (grade), TNM stage, and Ragnum hypoxia score (hypoxia score), were included in the univariate and multivariate CPHR analyses of the primary cohort. Factors with a $P$-value $<0.05$ both in the univariate and multivariate CPHR analyses were confirmed as independent prognostic variables and then used to establish a novel nomogram by using the package "rms" in
$\mathrm{R}$ software. To evaluate the predictive ability of the novel nomogram, a calibration plot was constructed and AUC in ROC analysis and $\mathrm{C}$-index were calculated. All analyses were implemented for the primary and internal validation cohorts.

\section{Functional enrichment analysis}

Three online databases, including TargetScan, miRTarBase, and miRDB, were used to predict the potential target genes of prognostic miRNAs. The overlapping miRNA target genes predicted by these three databases were undergo to GO analysis and KEGG pathway enrichment analysis using DAVID (http://david.ncifcrf.gov/ ) refer to previous description [34]. $P<0.05$ was considered the cut-off value.

\section{Abbreviations \\ HNSCC: Head and neck squamous cell carcinoma; miRNA: microRNA; GO: Gene Ontology; KEGG: Kyoto Encyclopedia of Genes and Genomes; DEM: Differentially expressed miRNA; OS: Overall survival; TCGA: The Cancer Genome Atlas; CPHR: Cox proportional hazard regression; OSCC: Oral squamous cell carcinoma}

\section{Acknowledgements}

Not applicable.

\section{Authors' contributions}

YFL, PWL and DLC conceived and designed the experiment; YSH, LMZ, ZGL and $Y W$ designed the research. YSH and LZG performed the experiments. LMZ, ZGL and YQX analyzed the data. YSH, YFL and PWL wrote the paper with contributions from all the authors. All authors have read and approved the manuscript

\section{Funding}

This work was supported by the National Natural Science Foundation of China (No. 81700512).

Availability of data and materials

The datasets used and/or analyzed during the current study are available from the corresponding author on reasonable request.

Ethics approval and consent to participate

Not applicable.

Consent for publication

Not applicable.

\section{Competing interests}

The authors declared no conflicts of interest.

\section{Author details}

${ }^{1}$ The First Affiliated Hospital, Guangzhou University of Chinese Medicine, No. 12 Airport Road, Baiyun District, Guangzhou 510407, China. ${ }^{2}$ Department of Oral and Maxillofacial Surgery, Guangdong Provincial Key Laboratory of Stomatology, Guanghua School of Stomatology, Sun Yat-Sen University, 56 Lingyuanxi Road, Guangzhou, China. ${ }^{3}$ Department of Laboratory Medicine, Guangdong Second Provincial General Hospital, Guangzhou, China.

${ }^{4}$ Department of Pediatrics, Shenzhen Hospital of Integrated Traditional Chinese and Western Medicine, Shenzhen, China. ${ }^{5}$ Guangzhou First People's Hospital, Guangzhou 510000, China. 
Received: 6 June 2020 Accepted: 24 September 2020

Published online: 06 October 2020

\section{References}

1. Ferlay J, Soerjomataram I, Dikshit R, Eser S, Mathers C, Rebelo M, et al. Cancer incidence and mortality worldwide: sources, methods and major patterns in GLOBOCAN 2012. Int J Cancer. 2015;136(5):E359-86.

2. Leemans $C$, Braakhuis B, Brakenhoff $R$. The molecular biology of head and neck cancer. Nat Rev Cancer. 2011;11:9-22.

3. Castellsagué X, Alemany L, Quer M, Halec G, Quirós B, Tous S, et al. HPV involvement in head and neck cancers: comprehensive assessment of biomarkers in 3680 patients. J Natl Cancer Inst. 2016;108:1-12.

4. Kim L, King T, Agulnik M. Head and neck cancer: changing epidemiology and public health implications. Head Neck. 2010;24(10):915-9.

5. Gregory RI, Shiekhattar R. MicroRNA biogenesis and cancer. Cancer Res. 2005;65(9):3509-12.

6. Gambari R, Brognara E, Spandidos DA, Fabbri E, et al. Targeting oncomiRNAs and mimicking tumor suppressor miRNAs: new trends in the development of miRNA therapeutic strategies in oncology (review). Int J Oncol. 2016;49(1):5-32

7. Saliminejad K, Khorram Khorshid HR, Soleymani Fard S, Ghaffari SH, et al. An overview of microRNAs: biology, functions, therapeutics, and analysis methods. J Cell Physiol. 2019;234(5):5451-65.

8. Fadhil RS, Wei MQ, Nikolarakos D, Good D, Nair RG. Salivary microRNA miRlet-7a-5p and miR-3928 could be used as potential diagnostic bio-markers for head and neck squamous cell carcinoma. PLoS One. 2020;15(3):e221779.

9. Lin XJ, Liu H, Li P, Wang HF, Yang AK, Di JM, et al. miR-936 suppresses cell proliferation, invasion, and drug resistance of laryngeal squamous cell carcinoma and targets GPR78. Front. Oncol. 2020;10:60.

10. Liu C, Yu Z, Huang S, Zhao Q, Sun Z, Fletcher C, et al. Combined identification of three miRNAs in serum as effective diagnostic biomarkers for HNSCC. EBioMedicine. 2019;50:135-43.

11. Kovaříková J, Baranová I, Laco J, Rozkošová K, Vošmíková H, Vošmík M, et al. Deregulation of Selected MicroRNAs in Sinonasal Squamous Cell Carcinoma: Searching for Potential Prognostic Biomarkers. Folia Biol (Praha). 2019;65(3): 142-51

12. Shiah SG, Hsiao JR, Chang HJ, Hsu YM, Wu GH, Peng HY, et al. MiR-30a and miR-379 modulate retinoic acid pathway by targeting DNA methyltransferase 3B in oral cancer. J Biomed Sci. 2020;27(1):46.

13. Hao Y, Zhang D, Guo Y, Fu Z, Yu D, Guan G, et al. miR-488-3p sponged by circ-0000495 and mediated upregulation of TROP2 in head and neck squamous cell carcinoma development. J Cancer. 2020;11(11):3375-86.

14. Kimura S, Naganuma S, Susuki D, Hirono Y, Yamaguchi A, Fujieda S, et al. Expression of microRNAs in squamous cell carcinoma of human head and neck and the esophagus: miR-205 and miR-21 are specific markers for HNSC C and ESCC. Oncol. Rep. 2010;23(6):1625-33.

15. Pedersen NJ, Jensen DH, Lelkaitis G, Kiss K, Charabi BW, Ullum H, et al. MicroRNA-based classifiers for diagnosis of oral cavity squamous cell carcinoma in tissue and plasma. Oral Oncol. 2018;83:46-52.

16. Chang M, Yan P, Zhang B, Zhang G, Wang J, Ge H, et al. MicroRNA-769-5p promotes the growth of Glioma cells by targeting lysine methyltransferase 2A. Onco Targets Ther. 2019;12:9177-87.

17. Wang W, Zhao LJ, Tan YX, Ren H, Qi ZT. Identification of deregulated miRNAs and their targets in hepatitis B virus-associated hepatocellular carcinoma. World J. Gastroenterol. 2012;18(38):5442-53.

18. Wang W, Zhao LJ, Tan YX, Ren H, Qi ZT. MiR-138 induces cell cycle arrest by targeting cyclin D3 in hepatocellular carcinoma. Carcinogenesis. 2012;33(5): 1113-20.

19. Zhao JR, Cheng WW, Wang YX, Cai M, Wu WB, Zhang HJ. Identification of microRNA signature in the progression of gestational trophoblastic disease. Cell Death Dis. 2018;9(2):94

20. Zhang L, Chen T, Yan L, Xu H, Wang Y, Li Y, et al. MiR-155-3p acts as a tumor suppressor and reverses paclitaxel resistance via negative regulation of MYD88 in human breast cancer. Gene. 2019;700:85-95.

21. He $Y$, Shi $Y$, Liu R, Wang Z, Wang B, Li S, et al. PELI3 mediates pro-tumor actions of down-regulated miR-365a-5p in non-small cell lung cancer. Biol Res. 2019;52(1):24

22. Hou YY, Lee JH, Chen HC, Yang CM, Huang SJ, Liou HH, et al. The association between miR-499a polymorphism and oral squamous cell carcinoma progression. Oral Dis. 2015;21(2):195-206.
23. Wang M, Yang C, Liu X, Zheng J, Xue Y, Ruan X, et al. An upstream open reading frame regulates vasculogenic mimicry of glioma via ZNRD1-AS1/ miR-499a-5p/ELF1/EMI1 pathway. J Cell Mol Med. 2020;24(11):6120-36.

24. Gu X, Dong M, Liu Z, Yang J, Shi Y. MiR-499a-5p inhibits proliferation, invasion, migration, and epithelial-Mesenchymal transition, and enhances Radiosensitivity of cervical Cancer cells via targeting elF4E. Onco Targets Ther. 2020;13:2913-24.

25. Zhuang Z, Yu P, Xie N, Wu Y, Liu H, Zhang M, et al. MicroRNA-204-5p is a tumor suppressor and potential therapeutic target in head and neck squamous cell carcinoma. Theranostics. 2020;10(3):1433-53.

26. Nunez Lopez YO, Victoria B, Golusinski P, Golusinski W, Masternak M. Characteristic miRNA expression signature and random forest survival analysis identify potential cancer-driving miRNAs in a broad range of head and neck squamous cell carcinoma subtypes. Rep Pract Oncol Radiother. 2018:23(1):6-20.

27. Chen-Tsai CP, Colome-Grimmer M, Wagner RF. Correlations among neural cell adhesion molecule, nerve growth factor, and its receptors, TrkA, TrkB, TrkC, and p75, in perineural invasion by basal cell and cutaneous squamous cell carcinomas. Dermatol Surg. 2004;30(7):1009-16.

28. Kupferman ME, Jiffar T, El-Naggar A, Yilmaz T, Zhou G, Xie T, et al. TrkB induces EMT and has a key role in invasion of head and neck squamous cell carcinoma. Oncogene. 2010;29(14):2047-59.

29. Sasahira T, Ueda N, Yamamoto K, Bhawal UK, Kurihara M, Kirita T, et al. Trks are novel oncogenes involved in the induction of neovascularization, tumor progression, and nodal metastasis in oral squamous cell carcinoma. Clin. Exp. Metastasis. 2013;30(2):165-76.

30. Yilmaz T, Jiffar T, de la Garza G, Lin H, Milas Z, Takahashi Y, et al. Theraputic targeting of Trk supresses tumor proliferation and enhances cisplatin activity in HNSCC. Cancer biol. Ther. 2010;10(6):644-53.

31. Gkouveris I, Nikitakis NG, Aseervatham J, Ogbureke KUE. Interferon $\gamma$ suppresses dentin sialophosphoprotein in oral squamous cell carcinoma cells resulting in antitumor effects, via modulation of the endoplasmic reticulum response. Int. J. Oncol. 2018:53(6):2423-32.

32. Gkouveris I, Nikitakis NG, Aseervatham J, Ogbureke K. The tumorigenic role of DSPP and its potential regulation of the unfolded protein response and ER stress in oral cancer cells. Int J Oncol. 2018;53(4):1743-51.

33. Liu R, Zhang L, Yang J, Zhang X, Mikkelsen R, Son S, et al. HIV Protease Inhibitors Sensitize Human Head and Neck Squamous Carcinoma Cells to Radiation by Activating Endoplasmic Reticulum Stress. PLoS One. 2015;10(5): e125928.

34. Zheng Z, Wen Y, Nie K, Tang S, Chen X, Lan S, et al. Construction of a 13microRNA-based signature and prognostic nomogram for predicting overall survival in patients with hepatocellular carcinoma. Hepatol Res. 2020. https://doi.org/10.1111/hepr.13538.

\section{Publisher's Note}

Springer Nature remains neutral with regard to jurisdictional claims in published maps and institutional affiliations.

Ready to submit your research? Choose BMC and benefit from:

- fast, convenient online submission

- thorough peer review by experienced researchers in your field

- rapid publication on acceptance

- support for research data, including large and complex data types

- gold Open Access which fosters wider collaboration and increased citations

- maximum visibility for your research: over $100 \mathrm{M}$ website views per year

At $\mathrm{BMC}$, research is always in progress.

Learn more biomedcentral.com/submission 